\title{
ALGEBRAIC CHARACTERIZATIONS OF LOCALLY COMPACT GROUPS
}

\author{
JUAN J. FONT and SALVADOR HERNÁNDEZ
}

(Received 15 May 1995; revised 21 June 1996)

Communicated by P. G. Dodds

\begin{abstract}
Let $G_{1}, G_{2}$ be locally compact real-compact spaces. A linear map $T$ defined from $C\left(G_{1}\right)$ into $C\left(G_{2}\right)$ is said to be separating or disjointness preserving if $f \cdot g \equiv 0$ implies $T f \cdot T g \equiv 0$ for all $f, g \in C\left(G_{1}\right)$. In this paper we prove that both a separating map which preserves non-vanishing functions and a separating bijection which satisfies condition (M) (see Definition 4) are automatically continuous and can be written as weighted composition maps. We also study the effect of separating surjections (respectively injections) on the underlying spaces $G_{1}$ and $G_{2}$.

Next we apply the above results to give an algebraic characterization of locally compact Abelian groups, similar to the one given in [7] for compact Abelian groups in the presence of ring isomorphisms.

Finally, locally compact (not necessarily Abelian) groups are considered. We provide a sharpening of a result of Edwards and study the effect of onto (respectively injective) weighted composition maps on the groups $G_{1}$ and $G_{2}$.
\end{abstract}

1991 Mathematics subject classification (Amer. Math. Soc.): primary 47B38; secondary 43A20, 54C40.

\section{Introduction}

Let $G_{1}, G_{2}$ be completely regular Hausdorff spaces. $C\left(G_{i}\right)(i=1,2)$ denotes the algebra of all complex-valued continuous functions on $G_{i}$ equipped with the compactopen topology. $C^{*}\left(G_{i}\right)$ denotes the subset of all bounded functions of $C\left(G_{i}\right)$. If $G_{i}$ is also locally compact, then let $C_{0}\left(G_{i}\right)$ (respectively $C_{00}\left(G_{i}\right)$ ) be the Banach algebra of all complex-valued continuous functions on $G_{i}$ which are zero at infinity (respectively the normed algebra of all continuous functions with compact support).

The deduction of topological links between $G_{1}$ and $G_{2}$ from certain algebraic relationships between $C\left(G_{1}\right)$ and $C\left(G_{2}\right)$ has been widely studied in the literature.

Research partially supported by Generalitat Valenciana, (2223/94)

(C) 1997 Australian Mathematical Society 0263-61 15/97 \$A2.00+0.00 
The first result of this type is the well-known Banach-Stone theorem. However, we follow the direction of the following classical result: if a linear map $T$ is an algebra isomorphism between $C\left(G_{1}\right)$ and $C\left(G_{2}\right)$, then the real-compactifications of $G_{1}$ and $G_{2}$ must be homeomorphic ([14, pp. 115-118]). Moreover, as in the Banach-Stone theorem, $T$ is a weighted composition map, that is, $T f=a \cdot(f \circ h)$ where 'the weight' $a$ belongs to $C\left(G_{2}\right)$ and $h: G_{2} \rightarrow G_{1}$ is a continuous map. Indeed, $|a| \equiv 1$ whenever $T$ is an onto linear isometry and $a \equiv 1$ provided $T$ is an algebra isomorphism. In this paper we deal with a weaker algebraic connection between $C\left(G_{1}\right)$ and $C\left(G_{2}\right)$.

A linear map $T$ defined from $C\left(G_{1}\right)$ into $C\left(G_{2}\right)$ is said to be separating or disjointness preserving (also called a $d$-homomorphism) if $f \cdot g \equiv 0$ implies $T f \cdot T g \equiv 0$ for all $f, g \in C\left(G_{1}\right)$. Throughout this paper, $T$ will denote a separating map unless otherwise specified. Algebra homomorphisms, lattice homomorphisms, onto isometries, and bipositive or weighted composition operators, are all separating maps.

Disjointness preserving maps between general vector lattices were considered first by several authors; see for example $[1,2,5,10,15,21]$. A thorough study of operators preserving disjointness in the context of $C(K)$-modules can be found in [3].

Disjointness preserving maps were later considered in [6] for spaces of real or complex-valued continuous functions defined on a compact Hausdorff space with the name of separating maps. The main goal of these two papers and $[20,4,11,12]$ is to prove automatic continuity results for separating maps when $G_{i}(\mathrm{i}=1,2)$ is compact, real-compact, locally compact and a locally compact Abelian group respectively. As a consequence, certain topological links between the underlying spaces are deduced and weighted composition type representations for separating maps are obtained. Similar representations have also been obtained in, for example, [1, 5, 19], though in the presence of a continuity type assumption about $T$. Automatic continuity for separating maps in the context of vector-valued continuous functions is considered in [16]. It is important to remark that a separating map need not be continuous; indeed, Jarosz proved in [20] that, given two compact spaces $G_{1}$ (infinite) and $G_{2}$, there always exists a discontinuous separating map defined from $C\left(G_{1}\right)$ onto $C\left(G_{2}\right)$.

In Section 3, we prove that both a separating map which preserves non-vanishing functions and a separating bijection which satisfies condition (M) (see Definition 4) are automatically continuous and can be written as weighted composition maps (Theorem 1 and Theorem 2, (1)). We also study the effect of separating injections, surjections and bijections on the underlying spaces $G_{1}$ and $G_{2}$ (Theorem 1 and Theorem 2, (2)-(4)).

In Section 4, we apply the results of Section 3 to give an algebraic characterization of locally compact Abelian groups, similar to the one given in [7] for compact Abelian groups in the presence of ring isomorphisms.

In Section 5, locally compact (not necessarily Abelian) groups are considered and $C_{00}\left(G_{i}\right)$ is regarded as an algebra under both the pointwise multiplication and the 
convolution product. We provide (Theorem 3 ) a sharpening of a result of Edwards ([8, Theorem 2]) since isometric and bipositive bijections are nothing but separating maps. Finally, we study the effect of onto (respectively injective) weighted composition maps on $G_{1}$ and $G_{2}$ (Theorems 4 and 5).

\section{Preliminaries}

$\mathbb{N}$ (respectively $\mathbb{R}, \mathbb{C}$ ) stands for the set of all natural numbers (respectively real, complex numbers). If $G_{i}$ is locally compact, then $G_{i}^{*}$ denotes its Alexandroff compactification; that is, $G_{i}^{*}=G_{i} \cup\{\infty\}, \infty$ being an ideal point with a neighbourhood consisting of all sets in $G_{i}^{*}$ whose complement is compact in $G_{i}$. Let $c G_{i}$ denote any Hausdorff compactification of $G_{i}$. If $f \in C\left(G_{i}\right)$, the co-zero of $f$ is the set $\operatorname{coz}(f)=\left\{t \in G_{i}: f(t) \neq 0\right\}$ and $\operatorname{supp}(f)$ will denote the closure of $\operatorname{coz}(f)$. When $U$ is any subset of $G_{i}$, we denote by $\operatorname{int}(U)$ the interior of $U$ and by $\operatorname{cl}(U)$ the closure of $U$ in $G_{i}$. We let $f_{\mid U}$ stand for the restriction of $f$ to $U$, for any $f \in C\left(G_{i}\right)$, and 1 denotes the unit of $C\left(G_{i}\right)$; that is, $1(s)=1$ for every $s \in G_{i}$.

Finally, if $s \in G_{2}$, let $T^{t} s^{t}: C\left(G_{1}\right) \rightarrow \mathbb{C}$ be defined as $T^{t} s^{t}(f)=T f(s)$ for all $f \in C\left(G_{1}\right)$.

\section{Automatic continuity and representation of separating maps}

Throughout this section $G_{1}, G_{2}$ will be locally compact real-compact spaces. The following two definitions and Propositions 1, 2 and 3 follow the pattern given by Abramovich in [1] for similar results in the context of general vector lattices.

DEFINITION 1. Given $s \in G_{2}$, we denote by $\operatorname{supp} T^{t} s^{t}$ the set $\left\{t \in c G_{1}\right.$ : for any $c G_{1}$-neighbourhood $U$ of $t$, there exists $f \in C\left(G_{1}\right)$ such that $T f(s) \neq 0$ and $\left.\operatorname{coz}(f) \subseteq U \cap G_{1}\right\}$.

LEMMA 1. For every $s \in G_{2}$, supp $T^{t} s^{t}$ has only one element.

PROOF. This can be found essentially in [1, Proposition 3.1]. (See also [6] or [20].)

DEFINITION 2. The lemma above lets us define a mapping $h: G_{2} \rightarrow c G_{1}$, such that $h(s)=\operatorname{supp} T^{t} s^{t}$. We call $h$ the support map of T.

PROPOSITION 1. Let $U$ be an open subset of $c G_{1}$ and suppose that $f \in C\left(G_{1}\right)$. Then the following conditions hold:

(1) The support map $h$ of $T$ is continuous. 
(2) $f_{\mid U \cap G_{1}} \equiv 0$ implies that $T f_{\mid h^{-1}(U)} \equiv 0$.

(3) $h(\operatorname{coz}(T f)) \subset c l_{c G_{1}}(\operatorname{coz}(f))$.

(4) If $T$ is injective, then $h\left(G_{2}\right)$ is a dense subset of $c G_{1}$.

PROOF. This can be essentially found in [1, Proposition 3.1]. (See also [6] or [20].)

REMARK. Let $G_{20} \subseteq G_{2}$ be the subset of all points $s$ such that there exists $f_{s} \in$ $C\left(G_{1}\right)$ with $T f_{s}(s) \neq 0$. Throughout this article we will assume that $G_{20}=G_{2}$. Note that if $T$ is onto or preserves non-vanishing functions, then $G_{20}=G_{2}$.

PROPOSITION 2. If $s \in G_{2}$ and $T^{t} s^{t}$ is a continuous map, then $h(s) \in G_{1}$.

PROOF. Let us suppose that $T^{t} s^{t}$ is continuous and $h(s) \in\left(c G_{1} \backslash G_{1}\right)$. Let $\left\{K_{a}\right.$ : $a \in A\}$ be the family of all compact subsets of $G_{1}$. Since $h(s) \notin G_{1}$, let us consider, for all $a \in A$, an open neighbourhood $V_{a}$ of $h(s)$ such that $c l_{c G}\left(V_{a}\right) \cap K_{a}=\emptyset$. By the definition of $h(s)$, there exists $f_{a} \in C\left(G_{1}\right)$ such that $\operatorname{coz}\left(f_{a}\right) \subset V_{a}$ and $T f_{a}(s)=1$.

On the other hand, we can order A by taking, for $a, b \in A, a \leq b$ if $K_{a} \subset K_{b}$, so that $\left(f_{a}\right)_{a \in A}$ form a net in $C\left(G_{1}\right)$. It is clear that, for all $a \in A, f_{a \mid K_{a}} \equiv 0$ and $f_{b \mid K_{a}} \equiv 0$ if $b \geq a$, that is, the net $\left(f_{a}\right)_{a \in A}$ converges to 0 in the compact-open topology of $C\left(G_{1}\right)$. This contradicts the continuity of $T^{t} s^{t}$ since $T^{t} s^{t}\left(f_{a}\right)=T f_{a}(s)=1$ for all $a \in A$.

DEFINITION 3. We denote by $G_{2 c}$ the subset of $G_{2}$ consisting of all $s \in G_{2}$ such that $T^{t} s^{t}$ is continuous, and by $G_{2 d}$ the complement of $G_{2 c}$ in $G_{2}$.

PROPOSITION 3. The following statements are equivalent.

(1) The map $T^{t} s^{t}$ is continuous for every $s \in G_{2}$, that is, $G_{2}=G_{2 c}$.

(2) $T$ is a weighted composition map. Indeed, $T f(s)=T 1(s) \cdot f(h(s))$ for every $s \in G_{2}$ and $f \in C\left(G_{1}\right)$.

(3) $T$ is a continuous map.

Proof. (1) implies (2). This is in [6, Theorem 2.2].

(2) implies (3). Let us consider a net $\left(f_{\alpha}\right)$ in $C\left(G_{1}\right)$ converging to $f \in C\left(G_{1}\right)$ in the compact-open topology. Given a compact subset $K$ of $G_{2}$, it suffices to prove that the net $\left(T f_{\alpha}\right)$ converges to $T f$ on $K$.

Let $\epsilon>0$. As $h(K)$ is a compact subset of $G_{1}$, there exists $\alpha^{\prime}$ such that $\sup \{|T 1(s)|$. $\left.\left|f_{\alpha}(h(s))-f(h(s))\right|: s \in K\right\}<\epsilon$ for every $\alpha \geq \alpha^{\prime}$ because the map $T 1$ is bounded on $K$. Thus, $\sup \left\{\left|T f_{\alpha}(s)-T f(s)\right|: s \in K\right\}<\epsilon$ and this completes the proof.

(3) implies (1) is clear. 
PROPOSITION 4. For any separating map T, the subsets of Definition 3 have the following properties:

(1) $G_{2 c}$ is closed in $h^{-1}\left(G_{1}\right)$.

(2) $h\left(G_{2 d}\right)$ is a subset of limit points of $c G_{1}$.

PROOF. (1) From Proposition 2 we have $G_{2 c} \subseteq h^{-1}\left(G_{1}\right)$. Let us consider a net ( $\left.s_{\alpha}\right)$ in $G_{2 c}$ which converges to $s \in h^{-1}\left(G_{1}\right)$. By Proposition 3, $T f\left(s_{\alpha}\right)=T 1\left(s_{\alpha}\right) \cdot f\left(h\left(s_{\alpha}\right)\right)$ for every $\alpha$ and every $f \in C\left(G_{1}\right)$. Since $T 1, f \circ h$, and $T f$ are continuous mappings, it is clear that $T f(s)=T 1(s) \cdot f(h(s))$ for every $f \in C\left(G_{1}\right)$; that is, $s \in G_{2 c}$.

(2) Let us see that if $h(s) \in G_{1}$ is isolated in $G_{1}$, for some $s \in G_{2}$, then $T^{t} s^{t}$ is a continuous map; that is, $s \in G_{2 c}$. Given $f \in C\left(G_{1}\right)$, let us define the map $g=f(h(s)) \cdot 1$. As $f_{\mid\{h(s)\}} \equiv g_{\mid\{h(s)\}}$, we have that $T f(s)=T g(s)$ by Proposition 1 . Hence, $T^{t} s^{t}(f)=T 1(s) \cdot f(h(s))$ for every $f \in C\left(G_{1}\right)$, which implies that $T^{t} s^{t}$ is continuous.

Definition 4. We say that $T$ satisfies condition $(M)$ if $T\left(C_{00}\left(G_{1}\right)\right) \subset C^{*}\left(G_{2}\right)$.

PROPOSITION 5. If the separating map $T$ satisfies condition $(M)$, then $h\left(G_{2 d}\right) \cap$ $\operatorname{int}(K)$ is finite for every compact subset $K$ of $G_{1}$.

Proof. To prove that $h\left(G_{2 d}\right) \cap \operatorname{int}(K)$ is finite for every compact subset $K$ of $G_{1}$, let us suppose that there exists a sequence $\left(h\left(s_{n}\right)\right)$ of distinct elements of int $(K)$ such that $s_{n} \in G_{2 d}$ for every $n \in \mathbb{N}$. As $K$ is a normal space, by taking a subsequence if necessary, we can assume that $\left\{U_{n}\right\}$ is a pairwise disjoint sequence of open subsets of $K$ such that $h\left(s_{n}\right) \in U_{n} \subseteq K$ for every $n \in \mathbb{N}$. Let $V_{n}$ be a closed neighbourhood of $h\left(s_{n}\right)$ with $V_{n} \subset U_{n}$. Thus, there exists a map $K_{n} \in C_{00}\left(G_{1}\right)$ such that $0 \leq K_{n} \leq \mathrm{i}$, $K_{n_{V_{n}}} \equiv 1$ and $\operatorname{coz}\left(K_{n}\right) \subset U_{n}$ for each $n \in \mathbb{N}$.

On the other hand, since $T^{t} s_{n}^{t}$ is discontinuous, there exists a map $f_{n} \in C\left(G_{1}\right)$ with $\sup \left\{\left|f_{n}(t)\right|: t \in K\right\} \leq 1$ and such that $\left|T^{t} s_{n}^{t}\left(f_{n}\right)\right|=\left|T f_{n}\left(s_{n}\right)\right|>n^{3}$ for all $n \in \mathbb{N}$. Let us define the map

$$
g_{n}=\frac{1}{n^{2}} \cdot f_{n} \cdot K_{n}
$$

Since $K_{n} \equiv 1$ on $V_{n}$, we have

$$
\left|T g_{n}\left(s_{n}\right)\right|=\frac{1}{n^{2}} \cdot\left|T f_{n}\left(s_{n}\right)\right|>n .
$$

Consequently, $\left|T g_{n}\left(s_{n}\right)\right|>n$ for each $n \in \mathbb{N}$.

It is clear that $\left\|g_{n}\right\| \leq 1 / n^{2}$ since $K_{n_{\mid T \backslash K}} \equiv 0$. Thus, we can define the map $g:=$ $\sum_{n \in \mathbb{N}} g_{n}$. Given that $\operatorname{supp}(g) \subseteq \operatorname{cl}\left(\bigcup\left(\operatorname{supp}\left(g_{n}\right)\right)\right) \subset K$, we deduce that $g \in C_{00}\left(G_{1}\right)$. On the other hand, as the family $\left\{U_{n}\right\}$ is pairwise disjoint and $\operatorname{coz}\left(g_{n}\right) \subset U_{n}$ for all 
$n \in \mathbb{N}$, then we have that $\left.T g_{n}\right|_{h^{-1}\left(U_{m}\right)} \equiv 0$ for $n \neq m$. Thus, $\left|T g\left(s_{n}\right)\right|=\left|T g_{n}\left(s_{n}\right)\right|>n$ for every $n \in \mathbb{N}$, which is a contradiction since $T$ satisfies condition $(M)$.

PROPOSITION 6. Let $T$ be a separating bijection from $C\left(G_{1}\right)$ onto $C\left(G_{2}\right)$ which satisfies condition $(M)$. Then:

(1) $G_{2 c}$ is dense in $G_{2}$; indeed $h^{-1}\left(G_{1}\right)=G_{2 c}$.

(2) $h\left(G_{2}\right) \subseteq G_{1}$.

PROOF. (1) As $G_{2 c} \cup G_{2 d}=G_{2}$, then $h\left(G_{2 c}\right) \cup h\left(G_{2 d}\right)=h\left(G_{2}\right)$. Since $T$ is injective, $h\left(G_{2}\right)$ is dense in $c G_{1}$ by Proposition 1(4). Hence, given $t \in G_{1}$ and a compact neighbourhood $U$ of $t$, we have, by Propositions 4(2) and 5, $h\left(G_{2 c}\right) \cap U \neq \emptyset$. That is, $h\left(G_{2 c}\right)$ is dense in $c G_{1}$.

Let $s \in h^{-1}\left(G_{1}\right)$ such that $s \notin G_{2 c}$. By Proposition 4, there exists a closed subset $C$ of $G_{2}$ such that $G_{2 c}=C \cap\left(h^{-1}\left(G_{1}\right)\right)$. Clearly, $s \notin C$. Since $T$ is onto, let $f \in C\left(G_{1}\right)$ such that $f \not \equiv 0, T f(s)=1$ and $T f_{\mid C} \equiv 0$. This implies that $T f_{\mid G_{2 c}} \equiv 0$. Consequently, $f \equiv 0$ since, from the onto-ness of $T, T 1(s) \neq 0$ for all $s \in G_{2 c}$ (see Proposition 3(2)) and $h\left(G_{2 c}\right)$ is dense in $c G_{1}$. This contradiction proves that $G_{2 c}=h^{-1}\left(G_{1}\right)$. In like manner, we prove that $G_{2 c}=h^{-1}\left(G_{1}\right)$ is dense in $G_{2}$.

(2) Let $s_{0} \in G_{2}$ be such that $h\left(s_{0}\right) \in\left(c G_{1} \backslash G_{1}\right)$. From (1), there exists a net $\left(s_{\alpha}\right)$ in $G_{2 c}$ such that $\left(s_{\alpha}\right)$ converges to $s_{0}$. Thus, by the continuity of $h$, we have that the net $\left(h\left(s_{\alpha}\right)\right)$ converges to $h\left(s_{0}\right)$. Let $K$ be a compact subset of $G_{2}$ such that $\left\{\left(s_{\alpha}\right) \bigcup\left\{s_{0}\right\}\right\} \subseteq K$. Let us suppose first that $T 1\left(s_{0}\right)=0$. Then $\left(\left|T 1\left(s_{\alpha}\right)\right| \cdot\left|f\left(h\left(s_{\alpha}\right)\right)\right|\right)=$ $\left(\left|T f\left(s_{\alpha}\right)\right|\right)$ converges to $\left|T f\left(s_{0}\right)\right|$ for all $f \in C\left(G_{1}\right)$. Since $(f \circ h)$ is bounded on $K$, $\left(\left|T 1\left(s_{\alpha}\right)\right| \cdot\left|f\left(h\left(s_{\alpha}\right)\right)\right|\right)$ converges to 0 . This implies that $T f\left(s_{0}\right)=0$ for all $f \in C\left(G_{1}\right)$, which contradicts the onto-ness of $T$.

On the other hand, let us suppose that $T 1\left(s_{0}\right) \neq 0$. Without loss of generality, we can assume that $c G_{1}$ is $\beta G_{1}$, the Stone-Čech compactification of $G_{1}$. Since $G_{1}$ is real-compact, there exists $([23, \mathrm{p} .81])$ a function $f_{0} \in C\left(\beta G_{1}\right)$ such that $f_{0}(t) \in \mathbb{R}$ for every $t \in \beta G_{1}, f_{0}\left(h\left(s_{0}\right)\right)=0$ and $f_{0}(t)>0$ for every $t \in G_{1}$. Let $g_{0}:=\left(1 / f_{0}\right)$. It is clear that $g_{0} \in C\left(G_{1}\right)$. Furthermore, $\left(\left|g_{0}\left(h\left(s_{\alpha}\right)\right)\right|\right)$ converges to $(+\infty)$ since the net $\left(s_{\alpha}\right)$ belongs to $G_{2 c}$ and $\left\{\left(s_{\alpha}\right) \bigcup\left\{s_{0}\right\}\right\} \subseteq K$. This contradicts the fact that the net $\left(\left|T 1\left(s_{\alpha}\right) \cdot g_{0}\left(h\left(s_{\alpha}\right)\right)\right|\right)=\left(\left|T g_{0}\left(s_{\alpha}\right)\right|\right)$ converges to $\left|T g_{0}\left(s_{0}\right)\right| \in \mathbb{R}$.

THEOREM 1. If there exists a separating bijection $T$ from $C\left(G_{1}\right)$ onto $C\left(G_{2}\right)$ which satisfies condition $(M)$, then $T$ is automatically continuous (indeed, a weighted composition map) and $G_{1}$ and $G_{2}$ are homeomorphic.

PROOF. The automatic continuity of $T$ and its multiplicative representation follow from Propositions 3 and 6 . This and the onto-ness of $T$ yield easily the injectivity of the map $h$ (note that $T 1$ is non-vanishing on $G_{2}$ ). 
Let us prove that the inverse of $T, T^{-1}$, is also a separating bijection. Let $g_{1}$ and $g_{2}$ be two elements of $C\left(G_{2}\right)$ such that $\operatorname{coz}\left(g_{1}\right) \cap \operatorname{coz}\left(g_{2}\right)=\emptyset$. Let $f_{1}, f_{2} \in C\left(G_{1}\right)$ be such that $T f_{1}=g_{1}$ and $T f_{2}=g_{2}$. Since $T 1$ is non-vanishing on $G_{2}$, we deduce that $\operatorname{coz}\left(f_{1}\right) \cap \operatorname{coz}\left(f_{2}\right)=\emptyset$ on $h\left(G_{2}\right)$, which is dense in $G_{1}$. As a consequence, $\operatorname{coz}\left(f_{1}\right) \cap \operatorname{coz}\left(f_{2}\right)=\emptyset$.

We can now define the support map $k: G_{1} \rightarrow c G_{2}$ of $T^{-1}$. Like $h, k$ is continuous and its range is dense in $c G_{2}$. Let $f \in C\left(G_{1}\right)$ be such that $T f \in C_{00}\left(G_{2}\right)$. From the multiplicative representation of $T$, we deduce that $(f \circ h)$ is bounded on $G_{2}$, which implies that $f$ is bounded on $G_{1}$. Therefore $T^{-1}$ also satisfies condition $(M)$. Consequently, we can represent $T^{-1}$ as a weighted composition map and $k\left(G_{1}\right) \subseteq G_{2}$.

If $t \in G_{1}$ is an element of $h\left(G_{2}\right)$, that is, $h(s)=t$ for some $s \in G_{2}$, we will show that $k(t)=s$. If $k(h(s)) \neq s$, then there exist disjoint compact neighbourhoods $U$ and $V$ of $k(h(s))$ and $s$ respectively. By the definition of $k$, there exists a function $f_{0} \in C\left(G_{2}\right)$ such that $\operatorname{coz}\left(f_{0}\right) \subseteq U$ and $T^{-1}\left(f_{0}\right)(h(s)) \neq 0$. This implies that $s \notin \operatorname{cl}\left(\operatorname{coz}\left(f_{0}\right)\right)$ and $k(h(s)) \in \operatorname{cl}\left(\operatorname{coz}\left(f_{0}\right)\right)$. Let $f_{1} \in C\left(G_{2}\right)$ such that $f_{1}(s) \neq 0$ and $f_{\mathbf{I}_{\mid \operatorname{coz}\left(f_{0}\right)}} \equiv 0$. Consequently, we have that $\operatorname{coz}\left(f_{1}\right) \cap \operatorname{coz}\left(f_{0}\right)=\emptyset$ and, since $T^{-1}$ is separating, $\operatorname{coz}\left(T^{-1}\left(f_{1}\right)\right) \cap \operatorname{coz}\left(T^{-1}\left(f_{0}\right)\right)=\emptyset$.

On the other hand, $\operatorname{coz}\left(T^{-1}\left(f_{0}\right)\right)$ is a neighbourhood of $h(s)$. If we take any $g \in C\left(G_{1}\right)$ such that $\operatorname{coz}(g) \subseteq \operatorname{coz}\left(T^{-1}\left(f_{0}\right)\right)$, then $\operatorname{coz}\left(f_{1}\right) \cap \operatorname{coz}(T g)=\emptyset$ because $T$ is separating. Consequently, $T g(s)=0$ since $f_{1}(s) \neq 0$. This contradicts the definition of $h$. In like manner, we obtain that $h^{-1}=k$, that is, $h$ is a homeomorphism of $G_{2}$ onto $G_{1}$.

REMARKS. Note that if $G_{2}$ is a pseudo-compact space, then condition (M) is redundant. Thus, we extend the result proved by Jarosz in [20]: Let $G_{1}$ and $G_{2}$ be compact spaces. If $T$ is a separating bijection of $C\left(G_{1}\right)$ onto $C\left(G_{2}\right)$, then $T$ is continuous, has a separating inverse and $G_{1}$ and $G_{2}$ are homeomorphic.

A close result is proved in [4] when $G_{1}$ and $G_{2}$ are real-compact and either the inverse of $T$ is separating or $G_{1}$ is zero-dimensional, though different techniques are used.

THEOREM 2. Let $T$ be a separating map from $C\left(G_{1}\right)$ into $C\left(G_{2}\right)$ which preserves non-vanishing functions. Then:

(1) $T$ is automatically continuous (indeed, a weighted composition map).

(2) If $T$ is onto, then there exists a closed subset $H_{1}$ of $G_{1}$ such that $H_{1}$ is homeomorphic to $G_{2}$.

(3) If $T$ is injective and preserves the functions with compact support, then $G_{1}$ is homeomorphic to a quotient of $G_{2}$.

(4) If $T$ is a bijection, then $G_{1}$ and $G_{2}$ are homeomorphic. 
Proof. (1) Let us first show that $h^{-1}\left(G_{1}\right)=G_{2 c}$. Suppose that there exists $s \in G_{2}$ such that $h(s) \in G_{1}$ and $T^{t} s^{t}$ is not continuous. Then, by Proposition 3 , there is some $f \in C\left(G_{1}\right)$ such that $f(h(s))=0$ but $T f(s) \neq 0$. Since $T 1(s) \neq 0$, we can choose $z \in C\left(G_{1}\right)$ such that $z \equiv 1$ on a neighbourhood $U$ of $h(s)$, while

$$
\sup \left\{|f z(t)|: t \in G_{1}\right\}<|T f(s)| /|T 1(s)| .
$$

Let

$$
g:=1-\frac{T 1(s) \cdot f z}{T(f z)(s)}
$$

Since $T(f z)(s)=T f(s)$ by Proposition 1(2), it follows that $g$ is non-vanishing, but $T g(s)=0$, which contradicts the non-vanishing-preserving property of $T$.

We next show that $h\left(G_{2}\right) \subseteq G_{1}$. Let us suppose that $h\left(s_{0}\right) \in\left(\beta G_{1} \backslash G_{1}\right)$ for some $s_{0} \in G_{2}$. Since $G_{1}$ is real-compact, there exists $([23, \mathrm{p} .81])$ a function $f_{0} \in C\left(\beta G_{1}\right)$ such that $f_{0}\left(h\left(s_{0}\right)\right)=0$ and $f_{0}$ never vanishes on $G_{1}$. Hence, by hypothesis, $T f_{0}\left(s_{0}\right) \neq$ 0 . Let $z_{0} \in C\left(\beta G_{1}\right)$ be such that $z_{0} \equiv 1$ on $U \cap G_{1}$, where $U$ is a neighbourhood of $h\left(s_{0}\right)$ in $\beta G_{1}$ and

$$
\sup \left\{\left|f_{0} z_{0}(t)\right|: t \in G_{1}\right\}<\left|T f_{0}\left(s_{0}\right)\right| /\left|T 1\left(s_{0}\right)\right| .
$$

If we take a function $g_{0}$ like $g$ above, we get the same contradiction.

By combining the preceding two paragraphs, we have $G_{2}=G_{2 c}$ and, therefore, $T$ is continuous from Proposition 3.

(2) From Propositions 2 and 3, we know that the range of $h$ is in $G_{1}$ and $T f(s)=$ $T 1(s) \cdot f(h(s))$ for all $f \in C\left(G_{1}\right)$ and all $s \in G_{2}$. In addition, $h$ is injective since $T$ is onto.

We will first prove that if $K$ is a compact subset of $G_{1}$, then $h^{-1}(K)$ is a compact subset of $G_{2}$. Otherwise, $h^{-1}(K)$ cannot be pseudo-compact since it is real-compact. Consequently, if $T 1$ is bounded on $h^{-1}(K)$, there is a sequence $\left(s_{n}\right)$ in $h^{-1}(K)$ and a function $g \in C\left(G_{2}\right)$ such that $\left|g\left(s_{n}\right)\right|>n \cdot\left|T 1\left(s_{n}\right)\right|$ for all $n \in \mathbb{N}$. On the other hand, if $T 1$ is not bounded on $h^{-1}(K)$, there is a sequence $\left(s_{n}\right)$ in $h^{-1}(K)$ such that $\left|T 1\left(s_{n}\right)\right|>n$. Thus, by taking $g=(T 1)^{2}$, we also have $\left|g\left(s_{n}\right)\right|>n \cdot\left|T 1\left(s_{n}\right)\right|$ for all $n \in \mathbb{N}$. Then, whether $T 1$ is bounded on $h^{-1}(K)$ or not, take $f \in C\left(G_{1}\right)$ such that $T f=g$. Since $T 1$ is non-vanishing, we deduce that $n \cdot\left|T 1\left(s_{n}\right)\right|<\left|T f\left(s_{n}\right)\right|=$ $\left|T 1\left(s_{n}\right)\right| \cdot\left|f\left(h\left(s_{n}\right)\right)\right|$. Hence, $f$ is not bounded on $K$, which is a contradiction.

Let us show now that $h\left(G_{2}\right)$ is a closed subset of $G_{1}$. Assume that $t \in c l_{G_{1}}\left(h\left(G_{2}\right)\right)$ and let $U$ be any compact neighbourhood of $t$. Thus, $h^{-1}(U)$ is a compact subset of $G_{2}$. Since $h^{-1}(U)=h^{-1}\left(U \cap h\left(G_{2}\right)\right)$, we obtain that $\left(U \cap h\left(G_{2}\right)\right)$ is a compact subset of $G_{1}$. That is, $t \in\left(U \cap h\left(G_{2}\right)\right)$.

Let $h^{*}: G_{2}^{*} \rightarrow\left(h\left(G_{2}\right)\right)^{*}$ be a map defined by the requirement that $h^{*}(\infty)$ be $\infty$ and $\left(h^{*}\right)_{\mid G_{2}} \equiv h$. To prove the continuity of $h^{*}$, it suffices to check that $h^{*}$ is continuous at 
$\infty$. Let $V$ be a neighbourhood of $\infty$ in $\left(h\left(G_{2}\right)\right)^{*}$. Hence there exists a compact subset $K$ of $h\left(G_{2}\right)$ such that $\left(h\left(G_{2}\right) \backslash K\right) \subseteq \mathrm{V}$. Since $h^{-1}(K)$ is compact in $G_{1},\left(G_{2} \backslash h^{-1}(K)\right)$ is a neighbourhood of $\infty$ and $h\left(G_{2} \backslash h^{-1}(K)\right) \subseteq\left(h\left(G_{2}\right) \backslash K\right) \subseteq \mathrm{V}$.

As a consequence, $h^{*}$ is a continuous bijection between $G_{2}^{*}$ and $\left(h\left(G_{2}\right)\right)^{*}$, which implies that $h$ is a homeomorphism of $G_{2}$ onto $h\left(G_{2}\right)$. It is now clear that $H_{1}:=h\left(G_{2}\right)$ satisfies the required conditions.

(3) By (1), we know that $T$ is automatically continuous, and thus we can write $T f(s)=T 1(s) \cdot f(h(s))$ for every $f \in C\left(G_{1}\right)$ and every $s \in G_{2}$.

Now let us prove that $h^{-1}(K)$ is compact for every compact subset $K$ of $G_{1}$. Take $f \in C_{00}\left(G_{1}\right)$ with $K \subseteq \operatorname{coz}(f)$. Then $h^{-1}(K) \subseteq h^{-1}\left(G_{1}\right)$. Furthermore, if $s \in h^{-1}(\operatorname{coz}(f)) \subset h^{-1}\left(G_{1}\right)$, then $f(h(s)) \neq 0$, which implies that $T f(s) \neq 0$. That is, $h^{-1}(K)$ is a closed subset of the compact set $\operatorname{supp}(T f)$.

As in the proof of (3), we obtain that the map $h^{*}: G_{2}^{*} \rightarrow G_{1}^{*}$ is continuous and, consequently, closed. In addition, we know that the range of $h$ is dense in $G_{1}$ since $T$ is injective. Hence, it is clear that $h^{*}$ is onto, closed, and continuous and so is $h$.

Therefore, by [9, Proposition 2.4.3], it is now evident that $G_{1}$ is homeomorphic to a quotient of $G_{2}$.

(4) We first show that $h\left(G_{2}\right)$ is $C$-embedded (see [14] for the definition) in $G_{1}$. Let $g_{0} \in C\left(h\left(G_{2}\right)\right)$. Let us consider the map $G: C\left(h\left(G_{2}\right)\right) \rightarrow C\left(G_{2}\right)$ defined by the requirement that $G(g)$ be $T 1 \cdot(g \circ h)$ for every $g \in C\left(h\left(G_{2}\right)\right)$. Since $T$ is onto, there exists a function $f_{0} \in C\left(G_{1}\right)$ such that $T f_{0}=G\left(g_{0}\right)$. Hence, $T 1(s) \cdot f_{0}(h(s))=T 1(s) \cdot g_{0}(h(s))$ for every $s \in G_{2}$. Since $T 1$ is non-vanishing, we have $f_{0} \circ h \equiv g_{0} \circ h$; that is, $f_{0 \mid h\left(G_{2}\right)} \equiv g_{0}$ and, thus, the function $f_{0} \in C\left(G_{1}\right)$ is the required extension.

Since $T$ is injective, we know the range of $h$ is dense in $G_{1}$. By combining this assertion, [14, Theorem 8.6], and the preceding paragraph, we deduce that $G_{1}=$ $v\left(h\left(G_{2}\right)\right)$, where $v\left(h\left(G_{2}\right)\right)$ stands for the real-compactification of $h\left(G_{2}\right)$.

Since $T$ is onto, we know that $h$ is injective. Let us consider $h^{-1}: h\left(G_{2}\right) \rightarrow G_{2}$, the inverse of $\mathbf{h}$. On the other hand, it is clear that $G$ is injective. Therefore, we can consider $G^{-1}: C\left(G_{2}\right) \rightarrow C\left(h\left(G_{2}\right)\right)$, the inverse of $\mathrm{G}$. It is also easy to verify that $G^{-1}(g)=g \circ h^{-1} / T 1$ for every $g \in C\left(G_{2}\right)$. Hence, $G^{-1}$ is also a separating map and its support map is $h^{-1}$. From Proposition 1 we have $h^{-1}$ is continuous. By [14, Theorem 8.6], $h^{-1}$ has a continuous extension $\left(h^{\prime}\right)^{-1}: G_{1} \rightarrow G_{2}$. As a consequence, $\left(h \circ\left(h^{\prime}\right)^{-1}\right)$ and the identity of $G_{1}$ coincide on $h\left(G_{2}\right)$ and, therefore on $G_{1}$, by the density of $h\left(G_{2}\right)$ in $G_{1}$. The proof of (4) is now complete.

REMARKS. (1) Theorem 2 is also valid when $G_{1}$ and $G_{2}$ are only real-compact spaces. However, if $G_{1}$ and $G_{2}$ are not real-compact, then Theorems 1 and 2 may fail. As an example, consider $G_{1}$ a pseudo-compact, not real-compact space and $v G_{1}$ its real-compactification, which is compact. It is easy to see that the natural 
isomorphism of $C\left(G_{1}\right)$ onto $C\left(v G_{1}\right)$ is a discontinuous separating bijection which preserves non-vanishing functions and satisfies condition (M).

However, let us recall (see [11]) that a separating bijection from $C_{0}\left(G_{1}\right)$ (respectively $C_{00}\left(G_{1}\right)$ ) onto $C_{0}\left(G_{2}\right)$ (respectively $\left.C_{00}\left(G_{2}\right)\right)$ is automatically continuous and induces a homeomorphism between the locally compact (not necessarily real-compact) spaces $G_{1}$ and $G_{2}$.

There remains open a complete answer to the following question: When is the inverse of a separating bijection defined from $C(X)$ onto $C(Y)(X, Y$ completely regular Hausdorff spaces) also separating?. Partial answers to this problem are then provided by Theorem 1 and Theorem 2 (4). In fact, such question was first proposed by Abramovich in the more general context of vector lattices. The first affirmative answer in this direction is the result (op. cit.) by Jarosz ([20]). Recently, Huijsmans and de Pagter ([18]) have proved the following: An invertible disjointness preserving operator from a Banach lattice onto a normed vector lattice has a disjointness preserving inverse and is norm bounded (=continuous).

(2) The following example shows that preserving functions with compact support is not a redundant hypothesis in Theorem 2(3): let us consider the map $T: C\left(\beta G_{2}\right) \rightarrow$ $C\left(G_{2}\right)$, where $\beta G_{2}$ denotes the Stone-Cech compactification of $G_{2}$, and such that $T f=f_{\mid G_{2}}$ for every $f \in C\left(\beta G_{2}\right)$. It is easy to verify that $T$ is a separating injection which preserves non-vanishing functions. However, $\beta G_{2}$ is not homeomorphic to a quotient of $G_{2}$.

(3) Finally, let us show, with an example due to Beckenstein and Narici, that there exist discontinuous separating injections: Let $G_{1}=[0,1]$ and $G_{2}=[0,1] \cup\{2\}$. For any $f \in C\left(G_{1}\right)$, let $T f(s)=(1-s) \cdot f(s)$ if $s \in[0,1]$ and $T f(2)=g(f)$, where $\mathrm{g}$ is a discontinuous separating functional defined like Example 3.6 in [6].

\section{Algebraic characterization of locally compact Abelian groups}

Throughout this section, $G_{1}$ and $G_{2}$ will be locally compact Abelian (LCA) groups. Let us recall that every locally compact group is real-compact. We say that a separating map $T$ of $C\left(G_{1}\right)$ into $C\left(G_{2}\right)$ is character preserving if given a character $\chi$ of $G_{1}$, that is, a complex-valued continuous homomorphism on $G_{1}$, then $T \chi$ is a character of $G_{2}$.

COROLLARY 1. If there exists a character preserving separating bijection $T$ from $C\left(G_{1}\right)$ onto $C\left(G_{2}\right)$ which either satisfies condition $(M)$ or preserves non-vanishing functions, then the LCA groups $G_{1}$ and $G_{2}$ are topologically isomorphic.

PROOF. By Theorems 1 and 2, we know that $T$ is a weighted composition map and $G_{1}$ and $G_{2}$ are homeomorphic. Hence, it suffices to check that the support map $h$ of $T$ is a homomorphism. 
Take $s_{1}, s_{2} \in G_{2}$ and let $\chi$ be any character of $G_{1}$. Hence, both $T \chi\left(s_{1} \cdot s_{2}\right)=$ $T 1\left(s_{1} \cdot s_{2}\right) \cdot \chi\left(h\left(s_{1} \cdot s_{2}\right)\right)$ and $T \chi\left(s_{1} \cdot s_{2}\right)=T \chi\left(s_{1}\right) \cdot T \chi\left(s_{2}\right)=T 1\left(s_{1}\right) \cdot \chi\left(h\left(s_{1}\right)\right) \cdot$ $T 1\left(s_{2}\right) \cdot \chi\left(h\left(s_{2}\right)\right)=T 1\left(s_{1} \cdot s_{2}\right) \cdot \chi\left(h\left(s_{1}\right)\right) \cdot \chi\left(h\left(s_{2}\right)\right)$. Since $T 1$ is non-vanishing, we have $\chi\left(h\left(s_{1} \cdot s_{2}\right)\right)=\chi\left(h\left(s_{1}\right)\right) \cdot \chi\left(h\left(s_{2}\right)\right)=\chi\left(h\left(s_{1}\right) \cdot h\left(s_{2}\right)\right)$ for every character $\chi$ of $G_{1}$, which implies that $h\left(s_{1} \cdot s_{2}\right)=h\left(s_{1}\right) \cdot h\left(s_{2}\right)$ and we are done.

COROLlary 2. Let $T$ be a separating map of $C\left(G_{1}\right)$ into $C\left(G_{2}\right)$ which preserves non-vanishing functions and characters.

(1) If $T$ is onto, then there exists a closed subgroup $H_{1}$ of $G_{1}$ such that $H_{1}$ is topologically isomorphic to $G_{2}$.

(2) If $T$ is injective and preserves functions with compact support, then there exists a closed subgroup $\mathrm{H}_{2}$ of $\mathrm{G}_{2}$ such that $G_{1}$ is topologically isomorphic to $\mathrm{G}_{2} / \mathrm{H}_{2}$.

PROOF. This follows from Theorem 2 (2) and Corollary 1.

(2) This follows from Theorem 2 (3) and Corollary $1, H_{2}$ being the kernel of the homomorphism $h$.

REMARK. If we consider the Bohr compactification $b G_{2}$ of $G_{2}$ instead of its StoneCech compactification in the remark following Theorem 2, then $T$ is also character preserving. Despite this, it is also evident that preserving functions with compact support is still not redundant.

\section{Algebraic characterization of locally compact groups}

Throughout this section, $G_{1}$ and $G_{2}$ are locally compact (not necessarily Abelian) groups. Let us also regard $C_{00}\left(G_{i}\right)(i=1,2)$ as an algebra under the convolution product $(f * g)(s)=\int_{G_{i}} f(z) \cdot g\left(z^{-1} \cdot s\right) d z$, integration being with respect to a fixed left Haar measure denoted by $d z$. Let $C_{00}^{+}\left(G_{i}\right)=\left\{f \in C_{00}\left(G_{i}\right): f \geq 0\right\}$.

DEFINITION 5. We say that a linear operator $T$ of $C_{00}\left(G_{1}\right)$ into $C_{00}\left(G_{2}\right)$ satisfies condition $(P)$ if given $s \in G_{2}$ and $f, g \in C_{00}^{+}\left(G_{1}\right)$ such that $T(f * g)(s)=0$, then $(T f * T g)(s)=0$.

It is evident that every convolution algebra homomorphism satisfies condition (P).

LEMMA 2. Let $T: C_{00}\left(G_{1}\right) \rightarrow C_{00}\left(G_{2}\right)$ be a map defined by the requirement that $T f=X(f \circ h)$ where $X$ is a non-vanishing scalar-valued continuous function defined on $G_{2}$ and $h$ is a continuous mapping from $G_{2}$ into $G_{1}$. Then the following statements are equivalent: 
(1) $h$ is a closed group homomorphism.

(2) $T$ satisfies condition $(P)$.

ProOF. (1) implies (2) Let us suppose that $f, g \in C_{00}^{+}\left(G_{1}\right)$ and $s \in G_{2}$ are such that $T(f * g)(s)=0$. Then, since $X$ does not vanish on $G_{2}$, we infer that $(f * g)(h(s))=0$; that is, $\int_{G_{1}} f(z) g\left(z^{-1} \cdot h(s)\right) d z=0$. Hence, from the continuity of $f$ and $g$, we have that $f(z) g\left(z^{-1} \cdot h(s)\right)=0$ for all $z \in G_{1}$.

On the other hand,

$$
\begin{aligned}
(T f * T g)(s) & =\int_{G_{2}} T f(y) T g\left(y^{-1} \cdot s\right) d y \\
& =\int_{G_{2}} X(y) X\left(y^{-1} \cdot s\right)(f \circ h)(y)(g \circ h)\left(y^{-1} \cdot s\right) d y .
\end{aligned}
$$

Since $h$ is a homomorphism, we deduce that

$$
(f \circ h)(y)(g \circ h)\left(y^{-1} \cdot s\right)=\left(f ( h ( y ) ) \left(g\left((h(y))^{-1} \cdot h(s)\right)=0\right.\right.
$$

for all $y \in G_{2}$. As a consequence, it is now clear that $(T f * T g)(s)=0$, as was to be proved.

(2) implies (1). Firstly let us see that $h$ is a closed mapping. As in the proof of Theorem 2 (3), we have that $h^{-1}(K)$ is compact for every compact subset $K$ of $G_{1}$. Then the map $h^{*}: G_{2}^{*} \rightarrow G_{1}^{*}$, defined as in Theorem $2(2)$, is continuous. Consequently, it is closed and so is $h$.

Next suppose that $h(s \cdot t) \neq h(s) \cdot h(t)$ for some $s, t \in G_{2}$. Then we can find open neighbourhoods $U$ and $V$ of $h(s)$ and $h(t)$ respectively such that $h(s \cdot t) \notin U \cdot V$. Let $f, g \in C_{00}^{+}\left(G_{1}\right)$ be such that $f(h(s))>0, g(h(t))>0, f$ vanishes outside $U$ and $g$ vanishes outside $V$.

Suppose firstly that $X$ is real-valued and let us assume, without loss of generality, that $X(s)>0$ and $X(t)<0$. Hence there exists a compact neighbourhood $W_{1}$ of the unit of $G_{2}$ such that $X_{\mid s W_{1}}>0$ and $X_{\mid W_{1} t}<0$. Let us take a compact neighbourhood $W$ of the unit of $G_{2}$ such that $W \subset \operatorname{int}\left(W_{1}\right)$. If $g_{h(s \cdot t)}$ stands for a function on $G_{1}$ defined by the requirement that $g_{h(s \cdot t)}(z)=g(z \cdot h(s \cdot t))$ for all $z \in G_{1}$, then it is easy to check that

$$
\int_{s W} X(y) X\left(y^{-1} \cdot s \cdot t\right)(f \circ h)(y)\left(g_{h(s \cdot t)} \circ h\right)\left(y^{-1}\right) d y=c<0 .
$$

Take now $k_{1} \in C_{00}^{+}\left(G_{1}\right)$ such that $0 \leq k_{1} \leq 1, k_{1}(h(s))=1$ and $k_{1}=0$ outside $h(s W)$. Similarly, let us consider $k_{2} \in C_{00}^{+}\left(G_{1}\right)$ such that $0 \leq k_{2} \leq 1, k_{2}(h(t))=1$ and $k_{2}=0$ outside $h(W t)$. Thus we have $\left(\left(k_{1} f\right) *\left(k_{2} g\right)\right)(h(s \cdot t))=0$. Since $T$ is a weighted composition map, $T\left(\left(k_{1} f\right) *\left(k_{2} g\right)\right)(s \cdot t)=0$. 
On the other hand and reasoning as above, we have

$$
\begin{aligned}
\left(T\left(k_{1} f\right)\right. & \left.* T\left(k_{2} g\right)\right)(s \cdot t) \\
& =\int_{G_{2}} X(y) X\left(y^{-1} \cdot s \cdot t\right)\left(k _ { 1 } ( h ( y ) ) \left(k _ { 2 } ( h ( y ^ { - 1 } \cdot s \cdot t ) ) \left(f ( h ( y ) ) \left(g_{h(s \cdot t)}\left(h\left(y^{-1}\right)\right) d y\right.\right.\right.\right. \\
& =\int_{s W} X(y) X\left(y^{-1} \cdot s \cdot t\right)\left(k _ { 1 } ( h ( y ) ) \left(k _ { 2 } ( h ( y ^ { - 1 } \cdot s \cdot t ) ) \left(f ( h ( y ) ) \left(g_{h(s \cdot t)}\left(h\left(y^{-1}\right)\right) d y\right.\right.\right.\right. \\
& \neq 0,
\end{aligned}
$$

which contradicts condition $(P)$. Assume now that $X$ is complex-valued, that is, $X=X_{1}+i X_{2}$. Hence

$$
\begin{aligned}
X(y) X\left(y^{-1} \cdot s \cdot t\right)= & \left(X_{1}(y) X_{1}\left(y^{-1} \cdot s \cdot t\right)-X_{2}(y) X_{2}\left(y^{-1} \cdot s \cdot t\right)\right) \\
& +i\left(X_{2}(y) X_{1}\left(y^{-1} \cdot s \cdot t\right)+X_{1}(y) X_{2}\left(y^{-1} \cdot s \cdot t\right)\right) .
\end{aligned}
$$

If we denote $\gamma(y)=X_{1}(y) X_{1}\left(y^{-1} \cdot s \cdot t\right)-X_{2}(y) X_{2}\left(y^{-1} \cdot s \cdot t\right)$, we will assume, without loss of generality, that $\gamma(s)>0$. As a consequence, there is a compact neighbourhood $W$ of the unit of $G_{2}$ such that for all $u \in s W$ and all $v \in W t$, $X_{1}(u) X_{1}(v)-X_{2}(u) X_{2}(v)>0$. The remainder of the proof follows from the same arguments as in the real-valued case.

THEOREM 3. Let $T$ be a separating bijection of $C_{00}\left(G_{1}\right)$ onto $C_{00}\left(G_{2}\right)$. Then the following statements are equivalent:

(1) There exists a topological isomorphism $h$ of $G_{2}$ onto $G_{1}$ such that $T f=X(f \circ h)$.

(2) $T$ satisfies condition $(P)$.

PROOF. According to Theorem 3 and Corollary in [11], we know that $T(f)=$ $X(f \circ h)$ where $X$ is a non-vanishing continuous map defined on $G_{2}$ and $h$ is a homeomorphism of $G_{2}$ onto $G_{1}$. Everything else is a consequence of Lemma 2 .

COROLlaRY 3. Let $T$ be a linear bijection of $C_{00}\left(G_{1}\right)$ onto $C_{00}\left(G_{2}\right)$ which is either bipositive or isometric. Then the following statements are equivalent:

(1) There exists a topological isomorphism h of $G_{2}$ onto $G_{1}$ such that $T f=X(f \circ h)$.

(2) $T$ satisfies condition $(P)$.

PROOF. From Theorem 3, it suffices to check that $T$ is a separating map in both cases. The isometric one is already proved in [11, Proposition 5].

Let us suppose that $T$ is a bipositive bijection. Given $s \in G_{2}$, let us define

$$
F_{s}=\left\{g \in C_{00}^{+}\left(G_{2}\right): g(s)>0\right\} \text {. }
$$


Fix $s_{0} \in G_{2}$. We shall show that $\bigcap_{T f \in F_{s_{0}}} \operatorname{supp}(f) \neq \emptyset$. Since, for every $f \in C_{00}^{+}\left(G_{1}\right)$, $\operatorname{supp}(f)$ is a compact subset of $G_{1}$, it is enough to prove that $\bigcap_{T f \in F} \operatorname{supp}(f) \neq \emptyset$ for every finite subset $F$ of $F_{s_{0}}$. There exist an open neighbourhood $V$ of $s_{0}$ and a positive constant $\lambda$ such that $T f_{\mid V}>\lambda$ for all $T f \in F$. Since $T$ is onto, we can choose $f_{1} \in C_{00}^{+}\left(G_{1}\right)$ such that, multiplying by a constant if necessary, $\left\|T f_{1}\right\| \leq \lambda$ and $\operatorname{supp}\left(T f_{1}\right) \subset V$. The positivity of the inverse of $T$ then implies that $\emptyset \neq \operatorname{supp}\left(f_{1}\right) \subset$ $\bigcap_{r \in F} \operatorname{supp}(f)$.

From the above arguments we derive the following: for every $s \in G_{2}$, there exists a subset $I_{s} \subset G_{1}$ such that, if $T f(s)>0$ for some $f \in C_{00}^{+}\left(G_{1}\right)$, then $t \in \operatorname{supp}(f)$ for all $t \in I_{s}$. That is, if for some $f \in C_{00}^{+}\left(G_{1}\right)$ and some open neighbourhood $U(t)$ of any $t \in I_{s}$ we have $f_{\mid U} \equiv 0$, then $T f(s)=0$.

Again fix $s_{0} \in G_{2}$ and let us take $f_{0} \in C_{00}^{+}\left(G_{1}\right)$ such that $f_{0}\left(t_{0}\right)=0$ for some $t_{0} \in I_{s_{0}}$. We will show that $T f_{0}\left(s_{0}\right)=0$. Let $\left(f_{\alpha}\right)$ be a net $C_{00}^{+}\left(G_{1}\right)$ such that $\left(f_{\alpha}\right)$ converges to $f_{0}$ and $f_{\alpha} \equiv 0$ on some open neighbourhood $V_{\alpha}$ of $t_{0}$ for all $\alpha$. Consequently, $T^{t} s_{0}^{t}\left(f_{\alpha}\right)=0$ for all $\alpha$.

Since $T^{t} s_{0}^{t}$ is a positive linear functional on $C_{00}\left(G_{1}\right)$, it is well known that, given a compact subset $K$ of $G_{1},\left|T^{t} s_{0}^{t}(f)\right| \leq C_{K} \cdot\|f\|, C_{K}$ a constant, for all $f \in C_{00}^{+}\left(G_{1}\right)$ whose support is contained in $K$. Without loss of generality, we can assume that $\operatorname{supp}\left(f_{\alpha}\right) \subset \operatorname{supp}\left(f_{0}\right)$ for all $\alpha$. Hence the net $\left(T^{t} s_{0}^{t}\left(f_{\alpha}\right)\right)$ converges to $T^{t} s_{0}^{t}\left(f_{0}\right)$, that is, $T^{t} s_{0}^{t}\left(f_{0}\right)=T f_{0}\left(s_{0}\right)=0$.

In general, if we choose $f_{0} \in C_{00}\left(G_{1}\right)$ such that $f_{0}\left(t_{0}\right)=0$ for some $t_{0} \in I_{s_{0}}$, then it is easy to check that $T f_{0}\left(s_{0}\right)=0$.

Finally, let $f_{1}, f_{2} \in C_{00}\left(G_{1}\right)$ be such that $f_{1} \cdot f_{2} \equiv 0$. Let us suppose that there exists $s_{0} \in G_{2}$ such that both $T f_{1}\left(s_{0}\right) \neq 0$ and $T f_{2}\left(s_{0}\right) \neq 0$. Then, since either $f_{1}(t)=0$ or $f_{2}(t)=0$ for any $t \in I_{s_{0}}$, we have either $T f_{1}\left(s_{0}\right)=0$ or $T f_{2}\left(s_{0}\right)=0$, which contradicts the above assumption and proves that $T$ is separating.

REMARK. In [8] (see also [24]), Edwards proved that if there exists a convolution algebra isomorphism of $C_{00}\left(G_{1}\right)$ onto $C_{00}\left(G_{2}\right)$ which is either bipositive or isometric, then $G_{1}$ and $G_{2}$ are isomorphic topological groups. Theorem 3 and its corollary above are then extensions of Edwards' results.

THEOREM 4. Let $T$ be a continuous separating map of $C_{00}\left(G_{1}\right)$ onto $C_{00}\left(G_{2}\right)$. Then the following statements are equivalent:

(1) There exists a topological isomorphism $h: G_{2} \rightarrow G_{1}$ such that $T f=X(f \circ h)$ (indeed, $G_{2}$ is topologically isomorphic to a closed subgroup of $G_{1}$ ).

(2) $T$ satisfies condition $(P)$.

PROOF. Let us suppose that $T$ satisfies condition $(P)$. Since $T$ is continuous, it can be written as a weighted composition map (see [11]); namely, $T f=X(f \circ$ 
$h$ ). By applying Lemma 2, we infer that $h$ is a closed injective continuous group homomorphism. Consequently, $h$ is a topological isomorphism.

The converse falls inmediately out of Lemma 2.

THEOREM 5. Let $T$ be a continuous separating injection of $C_{00}\left(G_{1}\right)$ into $C_{00}\left(G_{2}\right)$. Then the following statements are equivalent:

(1) There exists a topological homomorphism $h$ of $G_{2}$ onto $G_{1}$ such that $T f=$ $X(f \circ h)$ (indeed, $G_{1}$ is topologically isomorphic to a quotient of $\left.G_{2}\right)$.

(2) $T$ satisfies condition $(P)$.

PROOF. Let us suppose that $T$ satisfies condition $(P)$. Arguments like those in Corollary 2 and Theorem 4 show that $G_{1}$ is topologically isomorphic to $G_{2} / \operatorname{ker}(h)$. The converse follows from Lemma 2.

\section{References}

[1] Y. Abramovich, 'Multiplicative representation of disjointness preserving operators', Indag. Math. 45 (1983), 265-279.

[2] Y. Abramovich, A. I. Veksler and A. V. Koldunov, 'On operators preserving disjointness', Soviet Math. Dokl. 248 (1979), 1033-1036.

[3] Y.Abramovich, E. L. Arenson and A. K. Kitover, Banach $C(K)$-modules and operators preserving disjointness, Pitman Res. Notes Math. Ser. 277 (Longman, Harlow, 1993).

[4] J. Araujo, E. Beckenstein and L. Narici, 'On biseparating maps between real-compact spaces', $J$. Math. Anal. Appl. 192 (1995), 258-265.

[5] W. Arendt, 'Spectral properties of Lamperti operators', Indiana Univ. Math. J. 32 (1983), 199-215.

[6] E. Beckenstein, L. Narici and R. Todd, 'Automatic continuity of linear maps on spaces of continuous functions', Manuscripta Math. 62 (1988), 257-275.

[7] M. V. Deshpande and S. R. Ghorpade, 'Algebraic characterization of compact Abelian groups', Amer. Math. Monthly 98 (1991), 235-237.

[8] R. E. Edwards, 'Bipositive and isometric isomorphisms of some convolution algebras', Canad. $J$. Math. 17 (1965), 839-846.

[9] R. Engelking, General topology (Polish Scientific Publishers, Warszawa, 1977).

[10] W. A. Feldman and J. F. Porter, 'Operators on Banach lattices as weighted compositions', J. London Math. Soc. 33 (1986), 149-156.

[11] J. J. Font and S. Hernández, 'Separating maps between locally compact spaces', Arch. Math. 63 (1994), 158-165.

[12] _ 'Automatic continuity and representation of certain linear isomorphisms between group algebras', Indag. Math. 6 (1995), 397-409.

[13] I. Gelfand and A. Kolmogoroff, 'On the ring of continuous functions on a topological space', Dokl. Akad. Nauk SSSR 22 (1939), 11-15.

[14] L. Gillman and M. Jerison, Rings of continuous functions (Van Nostrand, Princeton, 1960).

[15] D. R. Hart, 'Some properties of disjointness preserving operators', Indag. Math. 47 (1985), 183197. 
[16] S. Hernández, E. Beckenstein and L. Narici, 'Banach-Stone theorems and separating maps', Manuscripta Math. 86 (1995), 409-416.

[17] E. Hewitt and K. A. Ross, Abstract harmonic analysis I (Springer, New York, 1963).

[18] C. B. Huijsmans and B. de Pagter, 'Invertible disjointness preserving operators', Proc. Edinburgh Math. Soc. 37 (1993), 125-132.

[19] J. E. Jamison and M. Rajagopalan, 'Weighted composition operators on $C(X, E)$ ', J. Operator Theory 19 (1988), 307-317.

[20] K. Jarosz, 'Automatic continuity of separating linear isomorphisms', Canad. Math. Bull. 33 (1990), $139-144$.

[21] B. de Pagter, 'A note on disjointness preserving operators', Proc. Amer. Math. Soc. 90 (1984), 543-549.

[22] W. Rudin, Fourier analysis on groups (Interscience Publishers, New York, 1962).

[23] M. D. Weir, Hewitt-Nachbin spaces, Stud. Math. Appl. 17 (North Holland, Amsterdam, 1975).

[24] K. Ylinen, 'Isomorphisms of spaces and convolution algebras of functions', Ann. Acad. Sci. Fenn. Ser. A Math. Dissertationes 510 (1972).

Departamento de Matemáticas

Universidad Jaume I

Campus Penyeta, E-12071

Castellón

Spain

e-mail: font@mat.uji.es, hernande@mat.uji.es

fax: 34-64-345847 